\title{
LncRNA SLC7A11-AS1 is Upregulated in Colorectal Cancer and Promotes the Proliferation of Cancer Cells by Suppressing the Maturation of miR-34a
}

\section{Peijiang Chang}

Qingdao Jiaozhou Central Hospital

Maosheng Wang ( $\square$ MaoshengWangYunnan@163.com )

Qujing Second People's Hospital https://orcid.org/0000-0002-1676-9135

\section{Research}

Keywords: colorectal cancer, SLC7A11-AS1, miR-34a, prognosis, maturation, proliferation

Posted Date: January 28th, 2021

DOl: https://doi.org/10.21203/rs.3.rs-155850/v1

License: (c) (1) This work is licensed under a Creative Commons Attribution 4.0 International License.

Read Full License 


\section{Abstract}

Background: LncRNA SLC7A11-AS1 is recently characterized critical player in cancer biology. We analyzed TCGA dataset and observed the upregulation of SLC7A11-AS1 in colorectal cancer (CRC). We therefore analyzed the role of SLC7A11-AS1 in CRC.

Methods: Paired CRC and non-tumor tissues were collected from $60 \mathrm{CRC}$ patients and expression of SLC7A11-AS1 in tissues was determined by RT-qPCR. The 60 CRC patients were followed up for 5 years to analyze the prognostic value of SLC7A11-AS1 for CRC. Correlations were analyzed by linear regression. The effects of SLC7A11-AS1 overexpression on the expression of miR-34a precursor and mature miR-34a were analyzed by RT-qPCR. Cell proliferation was analyzed by CCK-8 assay.

Result: SLC7A11-AS1 was upregulated in CRC and predicted poor survival. SLC7A11-AS1 and mature miR-34a were inversely correlated, while SLC7A11-AS1 was not significantly correlated with the precursor of miR-34a. In CRC cells, SLC7A11-AS1 overexpression resulted in the reduced level of mature miR-34a, but not miR-34a precursor. Moreover, SLC7A11-AS1 overexpression reduced the inhibitory effects of miR34a overexpression on cell proliferation.

Conclusion: SLC7A11-AS1 may promote the proliferation of cancer cells in CRC by suppressing the maturation of miR-34a.

\section{Background}

Colorectal cancer (CRC) as a type of malignancy originates from rectum or colon has been recognized as a common cancer for both incidence and mortality in clinical practices(1). It has been reported that CRC in 2018 affected 1800977 new cases, accounting for $10.0 \%$ of all new cancer cases, and caused 861663 deaths, which also account for $10.0 \%$ of all cancer deaths(2). It is estimated that more than $90 \%$ of patients with local CRC can survive 5 years after initial diagnosis $(3,4)$. However, metastasis to distant organs, such as lung and liver, is common(5). Once distant metastasis has occurred, no cure is available and the 5 -year overall survival rate will drop to below $50 \%(6)$. Therefore, novel therapeutic approaches are still needed.

Obesity, physical inactivity, smoking, alcohol abuse and aging are the main risk factors for $\mathrm{CRC}(7)$. Besides that, it has been well established that multiple molecular players are also involved in the pathogenesis of CRC(8-11). The increased understanding of the molecular mechanism of CRC facilitate the development of more effects treatments, such as targeted therapy that can be performed to suppress cancer progression by regulating related gene expression network $(8,10)$. Non-coding RNAs, such as miRNAs and IncRNAs, are not involved in protein synthesis but regulate gene expression to play their roles in cancer biology $(12,13)$. Therefore, IncRNAs and miRNAs are promising targets for cancer treatment. LncRNA SLC7A11-AS1 has been reported to be a critical player in gastric cancer and pancreatic cancer $(14,15)$, while its role in CRC is unknown. We analyzed TCGA dataset and observed the upregulation of SLC7A11-AS1 in CRC. In addition, our preliminary deep sequencing analysis revealed the 
inverse correlation between SLC7A11-AS1 and miR-34a, which is a well-established tumor suppressor(16). This study was therefore performed to analyze the interaction between SLC7A11-AS1 and miR-34a in CRC.

\section{Materials And Methods}

\section{Patients and tissue collections}

This study included 60 CRC patients (38 males and 22 females) at Qujing Second People's Hospital of Yunnan Province. Age of the patients ranged from 42 to 69 years (55.4t/-5.9 years). All the patients were newly diagnosed cases and patients complicated with other clinical disorders were excluded. No initiated therapy was observed before admission. This study was approved by the aforementioned hospital Ethics Committee. All patients signed informed consent. Prior to therapy, fine needle aspiration was performed to collect CRC and paired non-tumor tissues from all patients. All tissues were confirmed by histopathological test, followed by RNA extractions and RT-qPCR to detect gene expression.

\section{Treatment and follow-up}

According to AJCC staging system, the 60 CRC patients included 29 cases at stage I or II, and 31 cases at stage III or IV. Based on clinical stages and patients' health conditions, therapies, such as radio therapy, chemotherapy, surgical resection and their combinations, were performed. From the day of admission, the 60 patients were followed up for 5 years. Based on follow-up data, survival curves were plotted. The 60 CRC patients were excluded from the ones died of causes unrelated to CRC.

\section{CRC cells and transient transfections}

RKO and CR4 human CRC cell lines (ATCC, USA) were used. Cell culture medium was 90\% EMEM and $10 \%$ FBS. Cells were cultivated in a $95 \%$ humidity and $5 \% \mathrm{CO}_{2}$ incubator at $37^{\circ} \mathrm{C}$.

Backbone vector expressing SLC7A11-AS1 was established with pcDNA3.1 (Invitrogen). Mimic of miR34a and negative control (NC) miRNA were purchased from Sigma-Aldrich. RKO and CR4 cells (108) were transfected with $1 \mu \mathrm{g}$ expression vector or $40 \mathrm{nM}$ miRNA using Lipofectamine 2000 (Invitrogen). Control (C) cells were untransfected cells. To perform NC experiments, cells were transfected with either NC miRNA or empty pcDNA3.1 vector. Following transfections, cells were cultivated in fresh medium for $48 \mathrm{~h}$ prior to subsequent experiments.

\section{RNA preparations}

Paired tissue samples and non-tumor tissues, as well as RKO and CR4 cells were subjected to RNA extractions using RNAzol reagent (Sigma-Aldrich). DNase I (Invitrogen) was used to digest RNA samples for $2 \mathrm{~h}$ at $37^{\circ} \mathrm{C}$ to completely remove genomic DNA. RNA purity was determined by rations of OD260/280.

\section{RT-qPCRs}


RNA samples with an OD 260/280 ratio close to 2.0 were reverse transcribed into cDNA samples using SSRT IV system (Invitrogen). To determine the expression of SLC7A11-AS1, QuantiTect SYBR Green PCR Kit (Qiagen) was used to perform all qPCRs with 18S rRNA as internal control. The expression of miR-34a precursor and mature miR-34a were determined using All-in-One ${ }^{T M}$ miRNA qRT-PCR Detection Kit (Genecopoeia). All steps were completed according to manufacturer's instructions. Sequence-specific forward and reverse primers were used to determine the expression of miR-34a precursor. To determine mature miR-34a expression, miRNAs were added with poly (A), and ploy $(T)$ was used as reverse primer and sequence-specific forward primer was also used. Three technical replicates were performed in each experiment and $\mathrm{Ct}$ values of targeted genes were normalized using $2^{-\Delta \Delta C T}$ method.

\section{CCK-8 assay}

RKO and CR4 cells with transfections were subjected to CCK-8 assay. Briefly, 3000 cells in $0.1 \mathrm{ml}$ fresh medium were seeded onto each well of a 96-well plate. Cells were cultivated under the aforementioned conditions. At $2 \mathrm{~h}$ prior to the measurement of OD values, CCK-8 solution (Sigma-Aldrich) was added to reach $10 \%$. OD values at $450 \mathrm{~nm}$ were measured every $24 \mathrm{~h}$ for a total of 4 days.

\section{Statistical analysis}

Average values of three technical replicates were used to express gene expression levels in paired tissues and data were compared by paired $t$ test. Mean+/-SD values of three independent replicates were used to express multiple transfection groups and were compared by ANOVA Tukey's test. Correlations were analyzed by linear regression. The 60 patients were divided into high and low SLC7A11-AS1 level groups $(n=30)$. The cutoff value was the median expression level of SLC7A11-AS1 in CRC tissues. Survival curves were plotted for both groups and were compared by log rank test. $P<0.05$ was deemed statistically significant.

\section{Results}

\section{Upregulation of SLC7A11-AS1 predicted poor survival of CRC patients}

TCGA dataset was explored using Gepia (http://gepia.cancer-pku.cn/) to analyze the expression of SLC7A11-AS1 in CRC. It was observed that expression level of SLC7A11-AS1 was higher in CRC tissues compared to non-tumor tissues (0.06 VS. 0.01). Expression of SLC7A11-AS1 in paired CRC and non-tumor tissues was determined by RT-qPCR. Compared to non-tumor tissues, CRC tissues exhibited significantly upregulated SLC7A11-AS1 (Fig.1A, p<0.001). Survival analysis showed that, compared to patients in low SLC7A11-AS1 level group, patients in high SLC7A11-AS1 level group exhibited significantly lower overall survival rate (Fig.1B).

SLC7A11-AS1 was inversely correlated with mature miR-34a, but not miR-34a precursor 
Expression of mature miR-34a and miR-34a precursor in CRC tissues was also determined by RT-qPCR. The correlations between SLC7A11-AS1 and mature miR-34a (Fig.2A) or miR-34a precursor (Fig.2B) were analyzed by linear regression. It was observed that SLC7A11-AS1 and mature miR-34a were inversely and significantly correlated across CRC tissues. In contrast, no significant correlation between SLC7A11-AS1 and miR-34a precursor was observed across CRC tissues.

\section{SLC7A11-AS1 overexpression resulted in the downregulation of mature miR-34a}

RKO and CR4 cells were transfected with SLC7A11-AS1 expression vector or miR-34a mimic to explore the relationship between them. Overexpression of SLC7A11-AS1 and miR-34a was confirmed by RT-qPCR (Fig.3A, p < 0.05). It was observed that SLC7A11-AS1 overexpression resulted in the downregulation of mature miR-34a (Fig.3B, p<0.05), but failed to significantly affect the expression of miR-34a precursor (Fig.3C). Moreover, overexpression of miR-34a showed no significant effects on the expression of SLC7A11-AS1 (Fig.3D).

\section{SLC7A11-AS1 overexpression promoted the proliferation of CRC cells through miR-34a}

The roles of SLC7A11-AS1 and miR-34a in regulating the proliferation of RKO and CR4 cells were analyzed by CCK-8 assay. SLC7A11-AS1 overexpression resulted in the increased proliferation of both RKO and CR4 cells, while miR-34a resulted in the decreased cell proliferation. Moreover, SLC7A11-AS1 overexpression reduced the inhibitory effects of miR-34a overexpression on cell proliferation $(p<0.05)$.

\section{Discussion}

This study explored the potential interaction between SLC7A11-AS1 and miR-34a in CRC. We found that SLC7A11-AS1 was upregulated in CRC and it could suppress the maturation of miR-34a to promote CRC cell proliferation.

Previous studies have shown that SLC7A11-AS1 have different expression patterns and plays different roles in different types of cancers(14, 15). SLC7A11-AS1 was downregulated in gastric cancer(14). Interestingly, SLC7A11-AS1 knockdown mediated the increased cell proliferation rate through ASK1p38MAPK/JNK signaling pathway, suggesting the tumor suppressive roles of SLC7A11-AS1 in gastric cancer(14). In contrast, SLC7A11-AS1 is overexpressed in pancreatic cancer and promotes the development of chemoresistance by suppressing the degradation of NRF2 mediated by SCF $\beta$-TRCP, suggesting its oncogenic functions(15). We analyzed TCGA dataset and observed the upregulation of SLC7A11-AS1 in CRC. In this study, the overexpression of SLC7A11-AS1 in CRC was also confirmed in 60 cases of CRC patients. Moreover, overexpression of SLC7A11-AS1 resulted in the increased proliferation of CRC cells. Therefore, SLC7A11-AS1 may play oncogenic roles in CRC.

At present, clinical treatment of CRC is still limited by the low early diagnostic rate, which is mainly due to the lack of sensitive biomarkers(17). In this study we proved that the high expression levels of SLC7A11AS1 were significantly correlated with the poor survival of CRC patients. Therefore, measuring the 
expression levels of SLC7A11-AS1 before therapy may assist the prognosis of CRC and selection of treatment strategies, thereby increasing the survival time. However, the accuracy of SLC7A11-AS1 as a prognostic factor for CRC remains to be further analyzed.

MiR-34a plays tumor suppressive roles in multiple cancers, such as $\mathrm{CRC}(16)$. In this study we confirmed the inhibitory effects of miR-34a on CRC cell proliferation. Interestingly, we showed that SLC7A11-AS1 overexpression in CRC cells resulted in the downregulation of mature miR-34a, but not miR-34a precursor. Therefore, SLC7A11-AS1 may suppress the maturation of miR-34a in CRC cells to play oncogenic roles. Although we did not perform in vivo animal model studies, we showed that SLC7A11-AS1 was inversely correlated with mature miR-34a, but not moR-34a precursor. Therefore, the regulation of the maturation of miR-34a may also be regulated by SLC7A11-AS1 in CRC patients.

\section{Conclusion}

In conclusion, SLC7A11-AS1 is overexpressed in CRC and it may suppress the maturation of miR-34a to promote CRC cell proliferation.

\section{Declarations}

\section{Ethical Approval and Consent to participate}

Informed consent was obtained from all individual participants included in the study. All producers were approved by Qujing Second People's Hospital of Yunnan Province

Ethics Committee. All patients signed informed consent. Procedures operated in this research were completed in keeping with the standards set out in the Announcement of Helsinki and laboratory guidelines of research in China.

\section{Consent to publish}

Not applicable.

\section{Availability of supporting data}

The data that support the findings of this study are available on request from the corresponding author.

The data are not publicly available due to their containing information that could compromise the privacy of research participants.

\section{Competing interests}

All other authors have no conflicts of interest. 
We declare that we do not have any commercial or associative interest that represents a conflict of interest in connection with the work submitted.

\section{Funding}

Not Applicable.

\section{Authors' contributions}

Peijiang Chang $₫$ Maosheng Wang: study concepts, literature research, clinical studies, data analysis, experimental studies, manuscript writing and review; Peijiang Chang®Maosheng Wang: study design, literature research, experimental studies and manuscript editing; Peijiang Chang: definition of intellectual content, clinical studies, data acquisition and statistical analysis; Maosheng Wang: data acquisition, manuscript preparation and data analysis, data acquisition and statistical analysis.

All authors have read and approve the submission of the manuscript.

\section{Acknowledgements}

Not Applicable.

\section{References}

1. Siegel R, Desantis C, Jemal A. Colorectal cancer statistics, 2014. CA: a cancer journal for clinicians. 2014;64(2):104-17.

2. Bray F, Ferlay J, Soerjomataram I, Siegel RL, Torre LA, Jemal A. Global cancer statistics 2018 : GLOBOCAN estimates of incidence and mortality worldwide for 36 cancers in 185 countries. CA: a cancer journal for clinicians. 2018;68(6):394-424.

3. Bosetti C, Levi F, Rosato V, Bertuccio P, Lucchini F, Negri E, et al. Recent trends in colorectal cancer mortality in Europe. International journal of cancer. 2011;129(1):180-91.

4. Shin A, Jung KW, Won YJ. Colorectal cancer mortality in Hong Kong of China, Japan, South Korea, and Singapore. World journal of gastroenterology. 2013;19(7):979-83.

5. Qiu M, Hu J, Yang D, Cosgrove DP, Xu R. Pattern of distant metastases in colorectal cancer: a SEER based study. Oncotarget. 2015;6(36):38658-66.

6. Gallagher DJ, Kemeny N. Metastatic colorectal cancer: from improved survival to potential cure. Oncology. 2010;78(3-4):237-48.

7. Johnson CM, Wei C, Ensor JE, Smolenski DJ, Amos Cl, Levin B, et al. Meta-analyses of colorectal cancer risk factors. Cancer causes \& control : CCC. 2013;24(6):1207-22.

8. Heinemann V, Douillard JY, Ducreux M, Peeters M. Targeted therapy in metastatic colorectal cancer -an example of personalised medicine in action. Cancer treatment reviews. 2013;39(6):592-601. 
9. Leggett B, Whitehall V. Role of the serrated pathway in colorectal cancer pathogenesis. Gastroenterology. 2010;138(6):2088-100.

10. Linnekamp JF, Wang X, Medema JP, Vermeulen L. Colorectal cancer heterogeneity and targeted therapy: a case for molecular disease subtypes. Cancer research. 2015;75(2):245-9.

11. Pritchard CC, Grady WM. Colorectal cancer molecular biology moves into clinical practice. Gut. 2011;60(1):116-29.

12. Farazi TA, Spitzer JI, Morozov P, Tuschl T. miRNAs in human cancer. The Journal of pathology. 2011;223(2):102-15.

13. Prensner JR, Chinnaiyan AM. The emergence of IncRNAs in cancer biology. Cancer discovery. 2011;1(5):391-407.

14. Luo Y, Wang C, Yong P, Ye P, Liu Z, Fu Z, et al. Decreased expression of the long non-coding RNA SLC7A11-AS1 predicts poor prognosis and promotes tumor growth in gastric cancer. Oncotarget. 2017;8(68):112530-49.

15. Yang Q, Li K, Huang X, Zhao C, Mei Y, Li X, et al. IncRNA SLC7A11-AS1 Promotes Chemoresistance by Blocking SCF( $\beta$-TRCP)-Mediated Degradation of NRF2 in Pancreatic Cancer. Molecular therapy Nucleic acids. 2020;19:974-85.

16. Slabáková E, Culig Z, Remšík J, Souček K. Alternative mechanisms of miR-34a regulation in cancer. Cell death \& disease. 2017;8(10):e3100.

17. Vatandoost N, Ghanbari J, Mojaver M, Avan A, Ghayour-Mobarhan M, Nedaeinia R, et al. Early detection of colorectal cancer: from conventional methods to novel biomarkers. Journal of cancer research and clinical oncology.

\section{Figures}

A

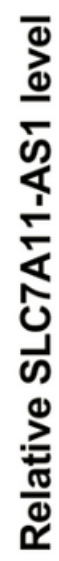

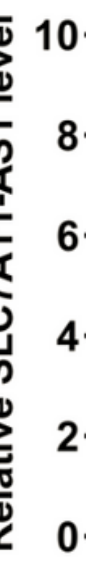
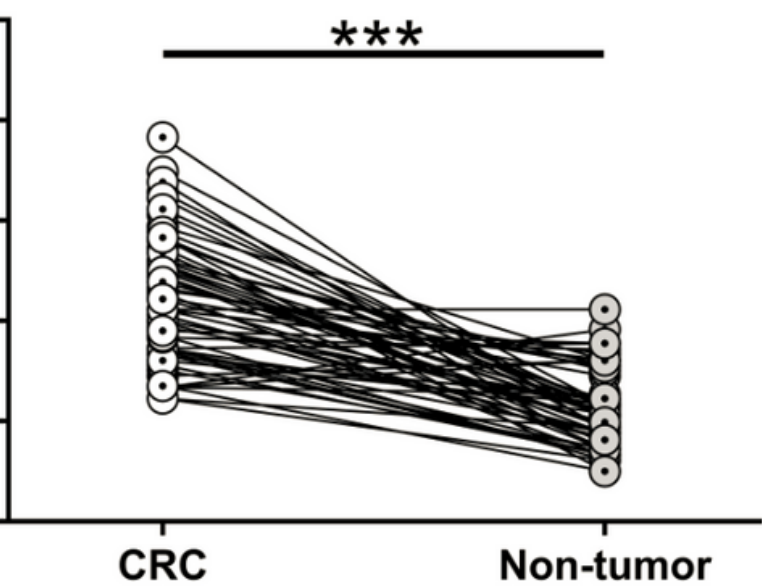

CRC

$* * *$
B

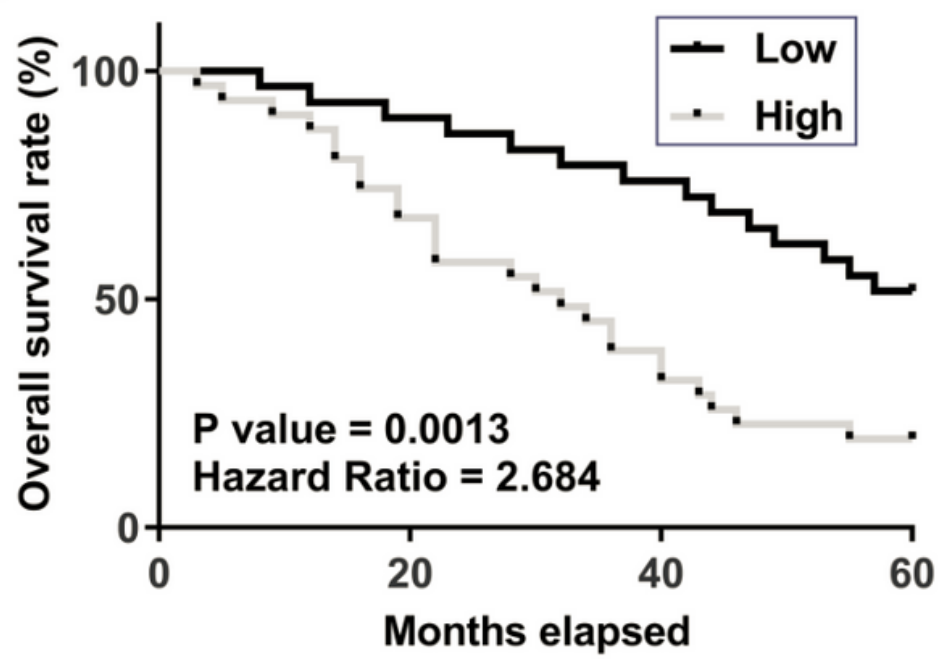

Figure 1 
Upregulation of SLC7A11-AS1 predicted poor survival of CRC patients Expression of SLC7A11-AS1 in paired CRC and non-tumor tissues was determined by RT-qPCR (A). Average values of three technical replicates were used to express gene expression levels in paired tissues and data were compared by paired $t$ test. ${ }^{* \star}, p<0.001$. The 60 patients were divided into high and low SLC7A11-AS1 level groups $(n=30)$. The cutoff value was the median expression level of SLC7A11-AS1 in CRC tissues. Survival curves were plotted for both groups and were compared by log rank test (B).

A

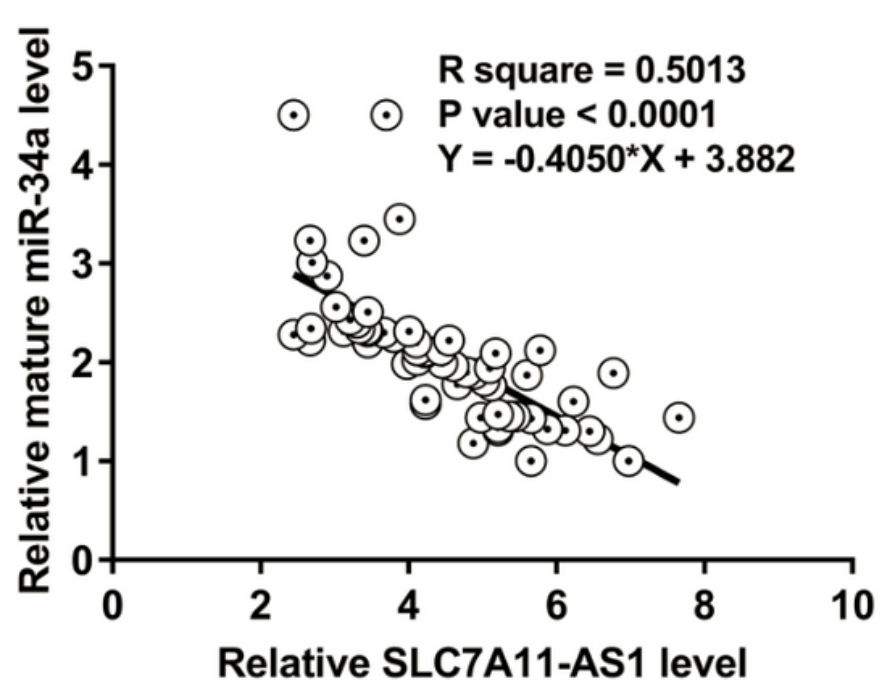

B

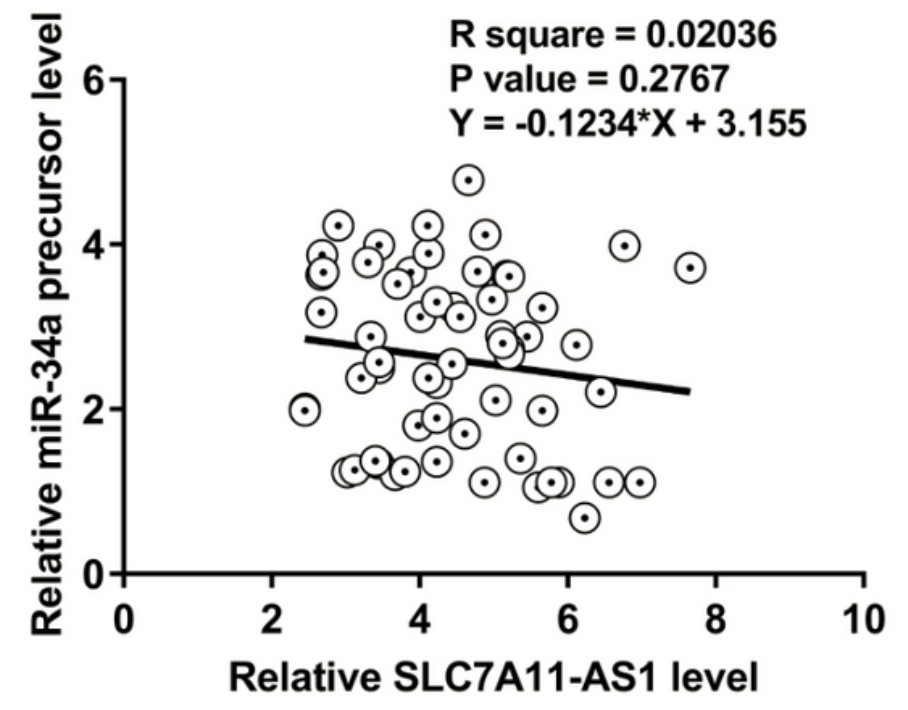

Figure 2

SLC7A11-AS1 was inversely correlated with mature miR-34a, but not miR-34a precursor Expression of mature miR-34a and miR-34a precursor in CRC tissues was also determined by RT-qPCR. The correlations between SLC7A11-AS1 and mature miR-34a (A) or miR-34a precursor (B) were analyzed by linear regression. 
A

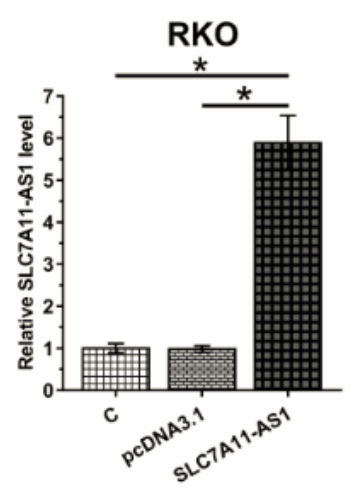

RKO

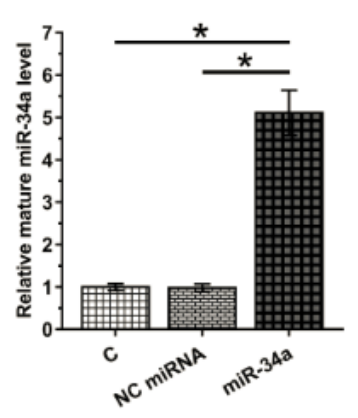

B

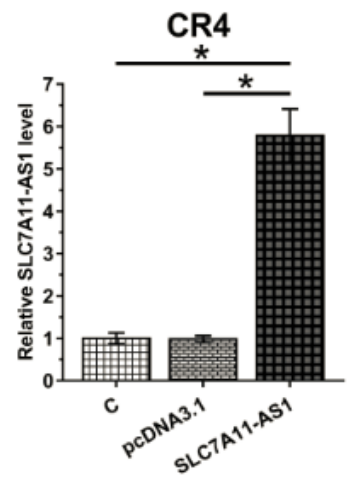

CR4

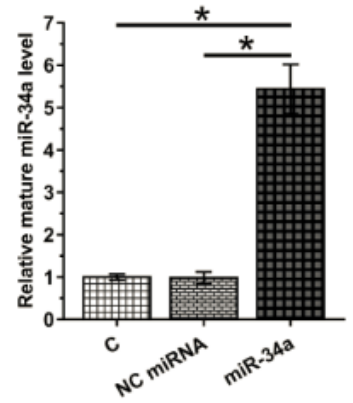

RKO

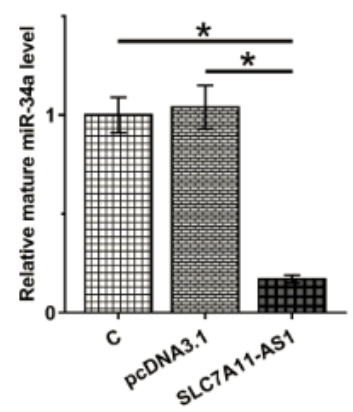

CR4

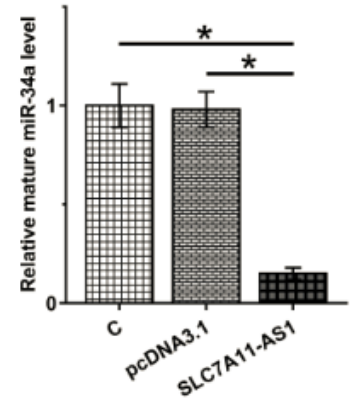

C

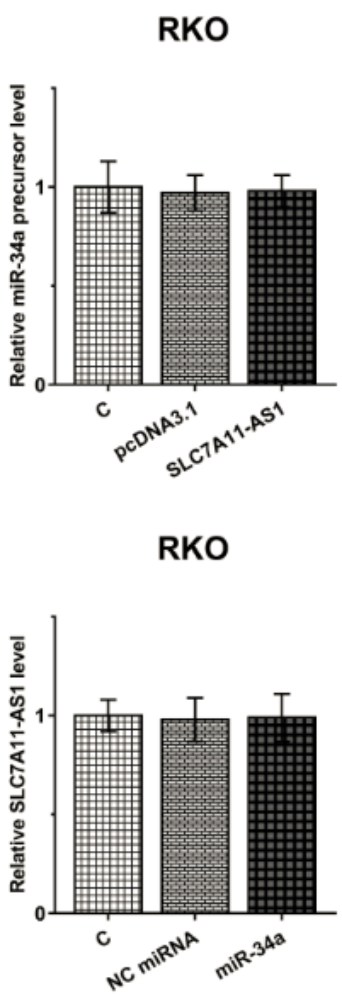

CR4

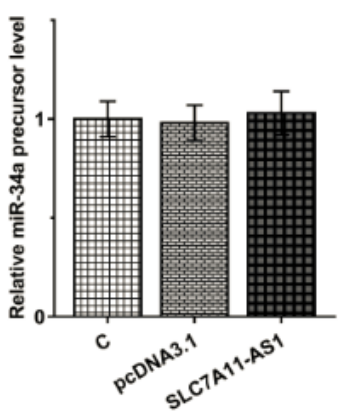

CR4

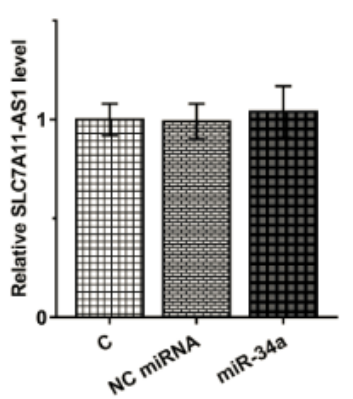

Figure 3

SLC7A11-AS1 overexpression resulted in the downregulation of mature miR-34a RKO and CR4 cells were transfected with SLC7A11-AS1 expression vector or miR-34a mimic to explore the relationship between them. Overexpression of SLC7A11-AS1 and miR-34a was confirmed by RT-qPCR (A). The effects of SLC7A11-AS1 overexpression on mature miR-34a (B) and miR-34a precursor (C), and the effects of miR34a overexpression on SLC7A11-AS1 (D) were also analyzed by RT-qPCR. Mean+/-SD values of three independent replicates were used to express multiple transfection groups. ${ }^{*}, p<0.05$.

\section{RKO}

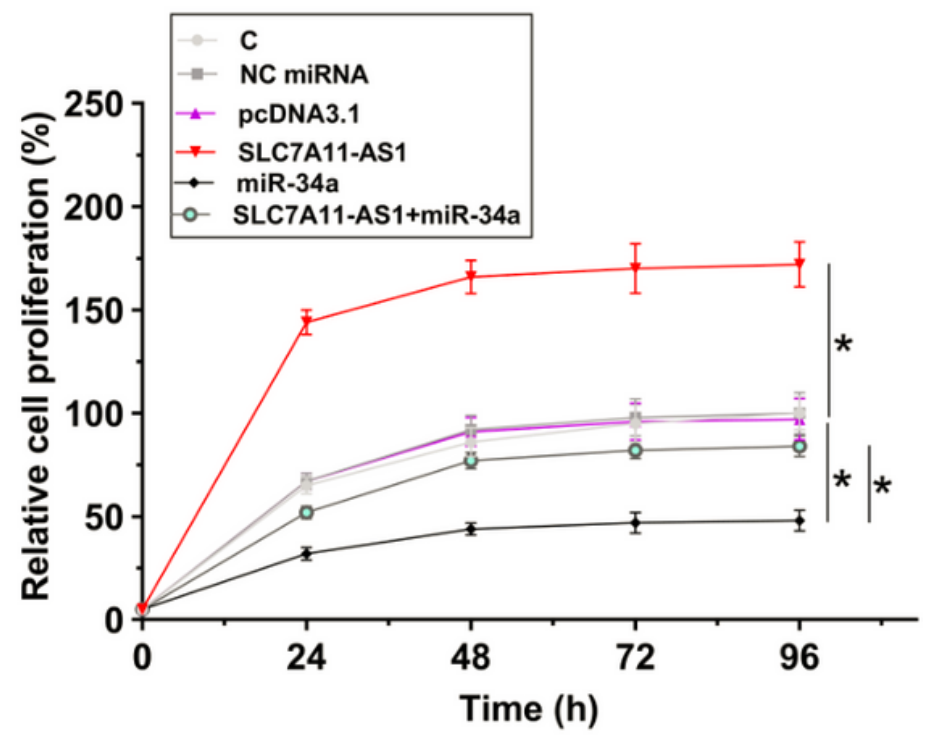

CR4

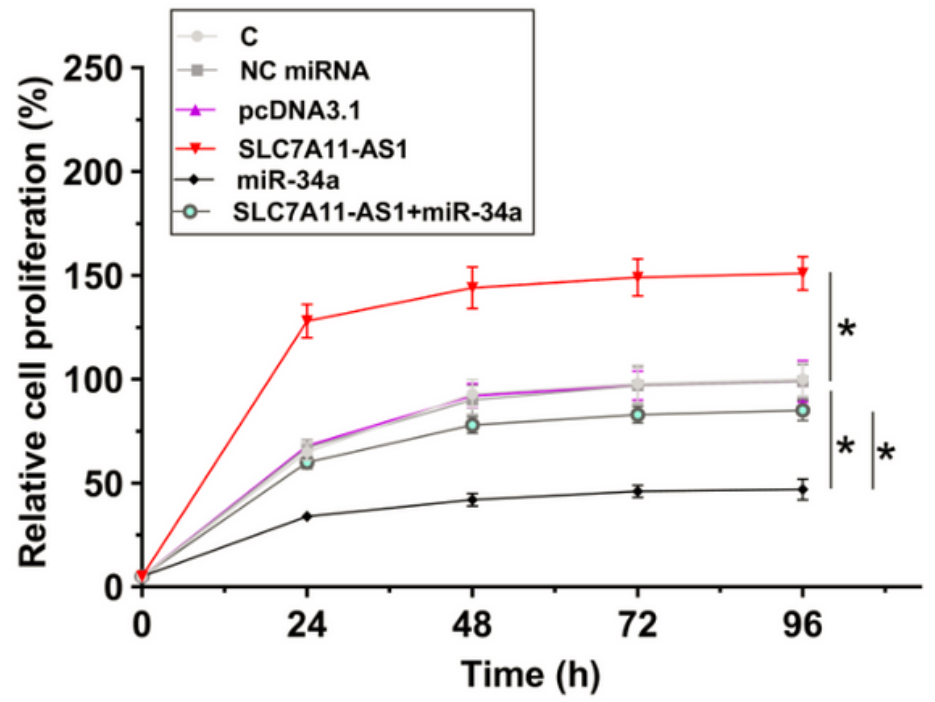

Figure 4 
SLC7A11-AS1 overexpression promoted the proliferation of CRC cells through miR-34a The roles of SLC7A11-AS1 and miR-34a in regulating the proliferation of RKO and CR4 cells were analyzed by CCK-8 assay. Mean+/-SD values of three independent replicates were used to express multiple transfection groups. ${ }^{\star}, \mathrm{p}<0.05$. 